\title{
On the asymptotic stability of discontinuous systems via averaging method
}

\author{
R. Gama ${ }^{\mathrm{a}}$, A. Guerman ${ }^{\mathrm{b}}$, G. Smirnov ${ }^{\mathrm{c}}$ \\ ${ }^{a}$ School of Technology and Management of Lamego \\ Av. Visconde Guedes Teixeira, 5100-074 Lamego, Portugal \\ ${ }^{b}$ Department of Electromechanical Engineering \\ University of Beira Interior \\ Calçada Fonte do Lameiro, 6201-001 Covilhã, Portugal \\ ${ }^{c}$ Centre of Mathematics of the Oporto University \\ Department of Mathematics and Applications, University of Minho \\ Campus de Gualtar, 4710-057 Braga, Portugal
}

\begin{abstract}
The averaging method is one of the most powerful methods used to analyse differential equations appearing in the study of nonlinear problems. The idea behind the averaging method is to replace the original equation by an averaged equation with simple structure and close solutions. A large number of practical problems leads to differential equations with discontinuous right-hand sides. In a rigorous theory of such systems, developed by Filippov, solutions of a differential equation with discontinuous right-hand side are regarded to be solutions to a special differential inclusion with upper semi-continuous right-hand side. Averaging method for such inclusions was studied by many authors under different and rather restrictive conditions on the regularity of the averaged inclusion. In this paper we prove natural extensions of Bogolyubov's first theorem and the Samoilenko-Stanzhitskii theorem to differential inclusions with an upper semi-continuous right-hand side. We prove that the solution set of the original differential inclusion is contained in a neighbourhood of the solution set of the averaged one. The extension of Bogolyubov's theorem concerns finite time intervals, while the extension of the Samoilenko-Stanzhitskii theorem deals with solutions defined on the infinite interval. The averaged inclusion is defined as a special upper limit
\end{abstract}

Email addresses: rgama@estgl.ipv.pt (R. Gama), anna@ubi.pt (A. Guerman), smirnov@math.uminho.pt (G. Smirnov) 
and no additional condition on its regularity is required.

Key words: Differential inclusions, Averaging method, Discontinuous right-hand side

2000 MSC: 34A60, 34C29, 34A36

\section{Introduction}

The averaging method is one of the most used methods to analyse differential equations of the form

$$
\dot{x}=\epsilon f(t, x),
$$

appearing in the study of nonlinear problems. The idea behind the averaging method is to replace the original equation by the averaged one:

$$
\dot{x}=\epsilon \bar{f}(x)=\epsilon \lim _{T \rightarrow \infty} \frac{1}{T} \int_{0}^{T} f(t, x) d t .
$$

This equation is simpler and has solutions close to the solutions of the original equation. A rigorous justification of the method is given by Bogolyubov's first theorem containing an estimate for the distance between the solutions of the exact and averaged systems on large time intervals [1]. The SamoilenkoStanzhitskii theorem [2, Theorem 2], a generalization of Bogolyubov's second theorem, shows that asymptotic stability of the zero equilibrium positon of averaged equation (2) implies that the solutions to original equation (1) are close to zero on the infinite time interval.

In many practical applications the function $f(t, \cdot)$ appearing in (1) is discontinuous (see, e.g., [3]), and the classical notion of solution and the classical averaging method cannot be used. For such systems Filippov proposed a generalized concept of solution, rewriting the problem (1) as a differential inclusion

$$
\dot{x} \in \epsilon F(t, x), \quad x(0)=x_{0},
$$

where $x \rightarrow F(\cdot, x)$ is an upper semi-continuous set-valued map obtained form $f(t, \cdot)$ by Filippov regularization [4, 5]. The use of this concept of solution makes it necessary to generalize the averaging method to differential inclusions. Many results extending Bogolyubov's first theorem to differential inclusions were obtained (see, e.g., [9, 10, 12, 13, 14, 15]). In the case of Lipschitzian differential inclusions the problem was completely solved by Plotnikov [9] and for inclusions with continuous right-hand side by Plotnikova [12] 
and Lakrib [16]. Averaging results for inclusions with upper semi-continuous right-hand side were obtained by Plotnikov [10] under conditions of Lipschitz continuity of the averaged inclusion and for inclusions with a piecewise Lipschitzian right-hand side. Some interesting results were proved by Donchev and Grammel [14] under one sided Lipschitz condition. Klimov [15] obtained a version of Bogolyubov's first theorem for inclusions satisfying a unilateral continuity condition.

In this paper we introduce an averaged differential inclusion suitable for discontinuous systems and prove extensions of Bogolyubov's first theorem and of the Samoilenko-Stanzhitskii theorem for upper semi-continuous differential inclusions, allowing one to extend the averaging method to the study of discontinuous dynamical systems.

Throughout this paper we denote by $\mathbb{R}^{n}$ the real $n$-dimensional space and by $\langle\cdot, \cdot\rangle$ and $|\cdot|$ the usual inner product and Euclidean norm, respectively. We use the notation $B=\left\{x \in \mathbb{R}^{n}|| x \mid \leq 1\right\}$ for the closed unit ball in $\mathbb{R}^{n}$. The interior, the convex hull, and the clusure of a subset $S \subset \mathbb{R}^{n}$ are denoted by int $S, \operatorname{co} S$, and $\mathrm{cl} S$, respectively. The Hausdorff distance between two sets $A_{1}, A_{2} \subset \mathbb{R}^{n}$ is defined as

$$
h\left(A_{1}, A_{2}\right)=\min \left\{h \geq 0 \mid A_{1} \subset A_{2}+h B, A_{2} \subset A_{1}+h B\right\} .
$$

The support function of a set $A \subset \mathbb{R}^{n}$ is denoted by $S(A, \psi)=\sup \{\langle a, \psi\rangle \mid$ $a \in A\}$. We denote by $\mathcal{S}_{[0, T]}\left(F, x_{0}\right)$ the set of solutions to the Cauchy problem $\dot{x} \in F(t, x), t \in[0, T], x(0)=x_{0}$, and by $\mathcal{R}_{[0, T]}\left(F, x_{0}\right)=\{x(T) \mid x(\cdot) \in$ $\left.\mathcal{S}_{[0, T]}\left(F, x_{0}\right)\right\}$ the reachability set. We use also the notations $\mathcal{S}_{[0, T]}(F, C)=$ $\bigcup_{x_{0} \in C} \mathcal{S}_{[0, T]}\left(F, x_{0}\right)$ and $\mathcal{S}_{[0, T]}(F)=\mathcal{S}_{[0, T]}\left(F, \mathbb{R}^{n}\right)$. The closed unit ball in the space of continuous functions $f:[0, T] \rightarrow \mathbb{R}^{n}$ with the uniform norm, $C\left([0, T], \mathbb{R}^{n}\right)$, is denoted by $\mathcal{B}$. The set of locally integrable functions $f$ : $\left[0, \infty\left[\rightarrow \mathbb{R}^{n}\right.\right.$ is denoted by $L_{1}^{\text {loc }}\left(\left[0, \infty\left[, \mathbb{R}^{n}\right)\right.\right.$.

\section{Averaged differential inclusion}

In this section we introduce an averaged differential inclusion suitable for discontinuous systems. Consider a differential inclusion

$$
\dot{x}(t) \in \epsilon F(t, x(t)) \text {. }
$$

Assume that there exists the limit

$$
\bar{F}(x)=\lim _{T \rightarrow \infty} \frac{1}{T} \int_{0}^{T} F(s, x) d s
$$


in the sense of the Hausdorff distance. Plotnikov, in his pioneering work [9], proved the estimate

$$
h\left(\mathcal{R}_{[0, T / \epsilon]}\left(\epsilon F, x_{0}\right), \mathcal{R}_{[0, T / \epsilon]}\left(\epsilon \bar{F}, x_{0}\right)\right)<\eta, \quad 0<\epsilon<\epsilon_{0}(\eta),
$$

provided that $F$ satisfies Lipschitz condition and limit (5) is uniform in $x$. If $F$ is discontinuous, estimate (6) cannot be obtained even for very simple system, as we can see from the following example.

\subsection{Example}

Consider the system

$$
\dot{x}=\epsilon f(t, x)
$$

where $x=\left(x_{1}, x_{2}\right) \in \mathbb{R}^{2}$ and

$$
f\left(t,\left(x_{1}, x_{2}\right)\right)= \begin{cases}v_{+}(t), & x_{2}>0 \\ v_{-}(t), & x_{2}<0\end{cases}
$$

with

$$
v_{+}(t)=\left(\sin \left(\frac{\pi}{4}(1+\cos t)\right),-\cos \left(\frac{\pi}{4}(1+\cos t)\right)\right)
$$

and

$$
v_{-}(t)=\left(\cos \left(\frac{\pi}{4}(1+\cos t)\right), \sin \left(\frac{\pi}{4}(1+\cos t)\right)\right) .
$$

Since $x \rightarrow f(\cdot, x)$ is discontinuous at the points $x=\left(x_{1}, 0\right)$, we apply Filippov's definition, obtaining the following differential inclusion:

$$
\dot{x} \in \epsilon F(t, x),
$$

where the set-valued map $F(t, x)$ is defined by

$$
F(t, x)=\left\{\begin{array}{cc}
f(t, x) & , x_{2} \neq 0 \\
I(t) & , x_{2}=0,
\end{array}\right.
$$

with $I(t)=\operatorname{co}\left\{v_{+}(t), v_{-}(t)\right\}$. This segment intersects the discontinuity line $x_{2}=0$. The intersection point is the velocity vector along this line,

$$
\left\{\left(f^{0}(t), 0\right)\right\}=I \cap\left\{\left(x_{1}, 0\right) \mid x_{1} \in \mathbb{R}\right\}
$$

where

$$
f^{0}(t)=\frac{1}{\sqrt{2}} \sec \left(\frac{\pi}{4} \cos t\right)
$$


The function $x(t)=\left(x_{1}(t), x_{2}(t)\right)$ satisfying

$$
\begin{cases}\dot{x}_{1}=\epsilon f^{0}(t), & x_{1}(0)=0 \\ \dot{x}_{2}=0, & x_{2}(0)=0\end{cases}
$$

is a unique solution of (7) belonging to the discontinuity line and satisfying zero initial condition. Observe that

$$
\bar{F}\left(\left(x_{1}, 0\right)\right)=\lim _{T \rightarrow \infty} \frac{1}{T} \int_{0}^{T} F\left(t,\left(x_{1}, 0\right)\right) d t \supset([\alpha, \beta], 0) \ni\left(f^{0}(t), 0\right)
$$

where $\alpha$ and $\beta$ are given by

$$
\alpha=\lim _{T \rightarrow \infty} \frac{1}{T} \int_{0}^{T} \min \left\{\cos \left(\frac{\pi}{4}(1+\cos t)\right), \sin \left(\frac{\pi}{4}(1+\cos t)\right)\right\} d t
$$

and

$$
\beta=\lim _{T \rightarrow \infty} \frac{1}{T} \int_{0}^{T} \max \left\{\cos \left(\frac{\pi}{4}(1+\cos t)\right), \sin \left(\frac{\pi}{4}(1+\cos t)\right)\right\} d t .
$$

Therefore we have

$$
\dot{\bar{x}}_{1} \in[\alpha, \beta], \quad \alpha<\beta .
$$

Thus, we see that differential inclusion (7) has a unique solution $x(t)$ belonging to the discontinuity line and satisfying zero initial condition, while the corresponding averaged one has a family of solutions.

This example shows that we can only guarantee a unilateral estimate

$$
\mathcal{R}_{[0, T / \epsilon]}\left(\epsilon F, x_{0}\right) \subset \mathcal{R}_{[0, T / \epsilon]}\left(\epsilon \bar{F}, x_{0}\right)+\eta B, \quad 0<\epsilon<\epsilon_{0}(\eta) .
$$

Since only a unilateral estimate is possible we do not need the existence of limit in the definition of averaged map (5). The limit can be substituted by the Kuratowski upper limit (see, e.g., [19]). Let $F: \mathbb{R} \times \mathbb{R}^{n} \rightarrow \mathbb{R}^{n}$ be a set-valued map. Set

$$
I\left(t_{1}, t_{2}, x, \delta\right)=\left\{\int_{t_{1}}^{t_{2}} v(t) d t \mid v(\cdot) \in L_{1}^{\mathrm{loc}}\left(\left[0, \infty\left[, \mathbb{R}^{n}\right), v(t) \in F(t, x+\delta B)\right\}\right.\right.
$$


We denote by $\bar{F}^{\delta}(x)$ the convex hull of the map

$$
\bar{\Phi}^{\delta}(x)=\limsup _{\theta \uparrow 1} \limsup _{T \rightarrow \infty} \frac{1}{(1-\theta) T} I(\theta T, T, x, \delta),
$$

and define the averaged differential inclusion as

$$
\dot{x} \in \bar{F}(x)=\bigcap_{\delta>0} \bar{F}^{\delta}(x) .
$$

Note that under Lipschitz condition this map coincides with (5), if the limit

$$
\overline{\bar{F}}(x)=\lim _{T \rightarrow \infty} \frac{1}{T} \int_{0}^{T} F(t, x) d t
$$

exists. Indeed, in this case we have

$$
I(\theta T, T, x, 0) \subset I(\theta T, T, x, \delta) \subset I(\theta T, T, x, 0)+l \delta(1-\theta) T B,
$$

where $l$ is the Lipschitz constant of the map $F(t, \cdot)$. From this we obtain

$$
\underset{\theta \uparrow 1}{\limsup } \limsup _{T \rightarrow \infty} \frac{1}{(1-\theta) T} \int_{\theta T}^{T} F(t, x) d t \subset \bar{\Phi}^{\delta}(x)
$$

and

$$
\bar{\Phi}^{\delta}(x) \subset \limsup _{\theta \uparrow 1} \limsup _{T \rightarrow \infty}\left(\frac{1}{(1-\theta) T} \int_{\theta T}^{T} F(t, x) d t+l \delta B\right)
$$

Passing to the limit in the equality

$$
\begin{gathered}
\frac{1}{(1-\theta) T} \int_{\theta T}^{T} S(F(t, x), \psi) d t \\
=\frac{1}{(1-\theta) T} \int_{0}^{T} S(F(t, x), \psi) d t-\frac{\theta}{(1-\theta) \theta T} \int_{0}^{\theta T} S(F(t, x), \psi) d t,
\end{gathered}
$$

holding for any $\psi \in \mathbb{R}^{n}$, we get

$$
\lim _{T \rightarrow \infty} \frac{1}{(1-\theta) T} \int_{\theta T}^{T} F(t, x) d t=\overline{\bar{F}}(x) .
$$

Combining this with $(11)$ and $\sqrt{12})$, we have $\overline{\bar{F}}(x) \subset \bar{\Phi}^{\delta}(x) \subset \overline{\bar{F}}(x)+l \delta B$. This implies the equality $\bar{F}(x)=\bar{F}(x)$.

Averaged inclusion (10) allows unilateral estimate even when limit (5) does not exist (cf. [11, 14]), as shows the following example. 


\subsection{Example}

Consider the differential inclusion

$$
\dot{x}(t) \in \epsilon F(t)=\epsilon\{\sin \log (t+1)\} .
$$

Obviously the limit

$$
\lim _{T \rightarrow \infty} \frac{1}{T} \int_{0}^{T} F(t) d t
$$

does not exist. However, $\bar{F} \equiv[-1 / \sqrt{2}, 1 / \sqrt{2}]$, and we have inclusion (8). Estimate (6), clearly, does not hold.

We show now that the map $\bar{F}$ cannot be substituted by the map $\bar{\Phi}^{0}$, if we have to guarantee the existence of solutions to the averaged differential inclusion.

\subsection{Example}

Consider the set-valued map $F:[0, \infty[\times \mathbb{R} \rightarrow \mathbb{R}$ given by

$$
F(t, x)= \begin{cases}\{1\}, & x \in]-\infty,-2 /(t+1)[, \\ \{-1-(t+1) x\}, & x \in[-2 /(t+1), 1 /(t+1)], \\ \{-2\}, & x \in] 1 /(t+1), \infty[\end{cases}
$$

Obviously we have

$$
\bar{\Phi}^{0}(x)= \begin{cases}\{1\}, & x<0 \\ \{-1\}, & x=0 \\ \{-2\}, & x>0\end{cases}
$$

The Cauchy problem $\dot{x} \in \bar{\Phi}^{0}(x), x(0)=0$, has no solution. The map

$$
\bar{F}(x)= \begin{cases}\{1\}, & x<0 \\ {[-2,1],} & x=0 \\ \{-2\}, & x>0\end{cases}
$$

is convex-valued and the Cauchy problem $\dot{x} \in \bar{F}(x), x(0)=0$, has a solution. 


\section{Main Results}

Let $F: \mathbb{R} \times \mathbb{R}^{n} \rightarrow \mathbb{R}^{n}$ be a set-valued map. Assume that the following conditions are satisfied:

(C1) $\operatorname{clco} F(t, x)=F(t, x)$, for all $(t, x) \in \mathbb{R} \times \mathbb{R}^{n}$;

(C2) the set-valued map $F(t, \cdot)$ is upper semicontinuous;

(C3) for any $x$ there exists measurable selection of $F(t, x)$, that is, there exists $f(t, x) \in F(t, x)$ such that $t \rightarrow f(t, x)$ is measurable for all $x$;

(C4) there exists a nonnegative $b(\cdot) \in L_{1}^{\text {loc }}([0, \infty[, \mathbb{R})$ such that $F(t, x) \subset$ $b(t) B$ for all $(t, x) \in\left[0,+\infty\left[\times \mathbb{R}^{n}\right.\right.$;

(C5) there exists the limit

$$
b=\lim _{T \rightarrow \infty} \frac{1}{T} \int_{0}^{T} b(t) d t
$$

Under this conditions we prove the following version of Bogolyubov's first theorem.

Theorem 3.1. Let $T>0$ and let $F: \mathbb{R} \times \mathbb{R}^{n} \rightarrow \mathbb{R}^{n}$ be a set-valued map satisfying conditions (C1) - (C5). Let $C \in \mathbb{R}^{n}$ be a compact set. Then for any $\eta>0$ there exists $\epsilon_{0}>0$ such that for any $\left.\epsilon \in\right] 0, \epsilon_{0}$ [ and any solution $x(\cdot) \in \mathcal{S}_{[0, T / \epsilon]}(\epsilon F, C)$, there exists a solution $\bar{x}(\cdot) \in \mathcal{S}_{[0, T / \epsilon]}(\epsilon \bar{F}, C)$ satisfying

$$
|x(t)-\bar{x}(t)|<\eta, \quad t \in[0, T / \epsilon] .
$$

Set

$$
G_{\epsilon}(\tau, y)=F(\tau / \epsilon, y) \text { and } G_{0}(y)=\bar{F}(y) .
$$

Next theorem is an extension of the Samoilenko-Stanzhitskii theorem.

Theorem 3.2. Let $F: \mathbb{R} \times \mathbb{R}^{n} \rightarrow \mathbb{R}^{n}$ be a set-valued map satisfying conditions (C1) - (C5). Assume that $y=0$ is an asymptotically stable equilibrium position of the differential inclusion $\dot{y} \in G_{0}(y)$. Then for any $\eta>0$ there exist $\epsilon_{0}>0$ and $\delta>0$ such that $\mathcal{S}_{[0, \infty[}\left(G_{\epsilon}, \delta B\right) \subset \eta \mathcal{B}$, whenever $\left.\epsilon \in\right] 0, \epsilon_{0}[$. 


\section{Approximation theorem}

In this section we show that the set of solutions to a non-autonomous differential inclusion

$$
\dot{x} \in F(t, x)
$$

with an upper semi-continuous with respect to $x$ right-hand side can be represented as an intersection of a decreasing sequence of set of solutions to Lipschitzian differential inclusions. This result is a generalization of Haddad's theorem from [17] and is the main tool used in the proof of the Bogolyubov theorem for discontinuous systems. A simplified version of this theorem appeared in [8].

Theorem 4.1. Let $F:\left[t_{0}, t_{1}\right] \times \mathbb{R}^{n} \rightarrow \mathbb{R}^{n}$ be a set-valued map satisfying Conditions (C1) - (C4). Then there exists a sequence of locally Lipschitzian set-valued maps $F_{k}:\left[t_{0}, t_{1}\right] \times \mathbb{R}^{n} \rightarrow \mathbb{R}^{n}, k=0,1, \ldots$, measurable in $t$ and satisfying the following conditions:

1. for any $k \geq 0,(t, x) \in\left[t_{0}, t_{1}\right] \times \mathbb{R}^{n}$ the inclusions $F_{k+1}(t, x) \subset F_{k}(t, x) \subset$ $b(t) B$ are fulfilled;

2. the inclusions $\mathcal{S}_{\left[t_{0}, t_{1}\right]}(F) \subset \mathcal{S}_{\left[t_{0}, t_{1}\right]}\left(F_{k}\right), k \geq 0$, hold;

3. for any pair $(t, x)$ and $\epsilon>0$ there exists a positive integer $M_{t, x, \epsilon}$ such that $F_{m}(t, x) \subset F(t, x)+\epsilon B$ for all $m>M_{t, x, \epsilon}$;

4. if a sequence of solutions $x_{k}(\cdot) \in \mathcal{S}_{\left[t_{0}, t_{1}\right]}\left(F_{k}\right)$ converges uniformly to a function $x(\cdot)$, then the inclusion $x(\cdot) \in \mathcal{S}_{\left[t_{0}, t_{1}\right]}(F)$ holds.

Note that this result does not contain any measurability condition with respect to both variables and cannot be deduced from the known approximation theorems (see, e.g., [6, 7]).

Proof of Theorem 4.1. Consider the set of points

$$
P=\left\{\left(\frac{\rho}{\sqrt{n}} r_{1}, \ldots, \frac{\rho}{\sqrt{n}} r_{n}\right) \mid r_{k}=0, \pm 1, \pm 2, \ldots, k=\overline{1, n}\right\} .
$$

where $\rho>0$. We denote the elements of $P$ by $x_{r}^{0}$, where $r \in \mathbb{Z}^{n}$ is a vector with integer components. Obviously $\left\{\left(x_{r}^{0}+\rho \operatorname{int} B\right)\right\}_{r \in \mathbb{Z}^{n}}$ is a locally finite covering of $\mathbb{R}^{n}$. Define the set-valued map $G_{r}^{0}(t)=\operatorname{co} F\left(t, x_{r}^{0}+2 \rho B\right)$. Let $V_{r}^{0}$ be the set of all integrable selections of $G_{r}^{0}(t)$. By conditions (C3) and (C4) this set is not empty. Let $\left\{v_{r, j}^{0}(\cdot)\right\}_{j=1}^{\infty} \subset V_{r}^{0}$ be a subset dense in the 
sense of $L_{1}$-norm. Set $\Phi_{r}^{0}(t)=\operatorname{cl} \operatorname{co}\left\{v_{r, j}^{0}(t) \mid j=\overline{1, \infty}\right\}$. The set-valued map $\Phi_{r}^{0}(\cdot)$ is measurable. Note that the function $v(\cdot) \in L_{1}\left(\left[t_{0}, t_{1}\right], R^{n}\right)$ satisfying the inclusion $v(t) \in G_{r}^{0}(t)$ also satisfies the inclusion $v(t) \in \Phi_{r}^{0}(t)$. In fact, suppose that a sequence $v_{r, j_{l}}^{0}(\cdot)$, satisfying $v_{r, j_{l}}^{0}(t) \in \Phi_{r}^{0}(t)$, converges to $v(\cdot)$, in $L_{1}\left(\left[t_{0}, t_{1}\right], R^{n}\right)$, as $l \rightarrow \infty$. Without loss of generality $v_{r, j_{l}}^{0}(t) \rightarrow v(t)$ for almost all $t \in\left[t_{0}, t_{1}\right]$. Since $\Phi_{r}^{0}(t)$ is a closed set, we have $v(t) \in \Phi_{r}^{0}(t)$.

Let $\left\{p_{r}^{0}(\cdot)\right\}_{r \in \mathbb{Z}^{n}}$ be a partition of unity subordinated to the covering $\left\{x_{r}^{0}+\right.$ $\rho \operatorname{int} B\}_{r \in \mathbb{Z}^{n}}$. Consider the set-valued map

$$
F_{0}(t, x)=\sum_{r \in \mathbb{Z}^{n}} p_{r}^{0}(x) \Phi_{r}^{0}(t)
$$

This set-valued map has closed convex images, is measurable in $t$ and Lipchitzian in $x$.

To define the set-valued map $F_{1}(t, x)$ we repeat the above procedure with $\rho / 3$ instead of $\rho$. As a result we obtain a locally finite open covering $\left\{x_{r}^{1}+\rho / 3 \operatorname{int} B\right\}_{r \in \mathbb{Z}^{n}}$ of $\mathbb{R}^{n}$, a corresponding partition of unity $\left\{p_{r}^{1}(\cdot)\right\}_{r \in \mathbb{Z}^{n}}$, set-valued maps $\left\{\Phi_{r}^{1}(\cdot)\right\}_{r \in \mathbb{Z}^{n}}$, and the set-valued map

$$
F_{1}(t, x)=\sum_{r \in \mathbb{Z}^{n}} p_{r}^{1}(x) \Phi_{r}^{1}(t)
$$

Let $x \in \mathbb{R}^{n}$. Define the sets

$$
I_{0}^{x}=\left\{r \in \mathbb{Z}^{n} \mid x \in x_{r}^{0}+\rho \operatorname{int} B\right\}
$$

and

$$
I_{1}^{x}=\left\{r \in \mathbb{Z}^{n} \mid x \in x_{r}^{1}+\frac{1}{3} \rho \operatorname{int} B\right\}
$$

Show that $x_{r_{1}}^{1}+(2 / 3) \rho \operatorname{int} B \subset x_{r_{0}}^{0}+2 \rho \operatorname{int} B$, whenever $r_{0} \in I_{0}^{x}$ and $r_{1} \in I_{1}^{x}$. Indeed, let $y \in x_{r_{1}}^{1}+(2 / 3) \rho \operatorname{int} B$, then we have

$$
\left|y-x_{r_{0}}^{0}\right| \leq\left|y-x_{r_{1}}^{1}\right|+\left|x_{r_{1}}^{1}-x\right|+\left|x-x_{r_{0}}^{0}\right| \leq \frac{2}{3} \rho+\frac{1}{3} \rho+\rho=2 \rho,
$$

that is, $G_{r_{1}}^{1}(t) \subset G_{r_{0}}^{0}(t)$ and, consequently, $\Phi_{r_{1}}^{1}(t) \subset \Phi_{r_{0}}^{0}(t)$. For all $r_{1} \in I_{1}^{x}$ we have

$$
\Phi_{r_{1}}^{1}(t) \subset \sum_{r \in I_{0}^{x}} p_{r}^{0}(x) \Phi_{r}^{0}(t)=\sum_{r \in \mathbb{Z}^{n}} p_{r}^{0}(x) \Phi_{r}^{0}(t)=F_{0}(t, x)
$$


and, therefore,

$$
F_{1}(t, x)=\sum_{r \in \mathbb{Z}^{n}} p_{r}^{1}(x) \Phi_{r}^{1}(t)=\sum_{r \in I_{1}^{x}} p_{r}^{1}(x) \Phi_{r}^{1}(t) \subset F_{0}(t, x) .
$$

Consider the sequence $\rho_{k}=(1 / 3)^{k} \rho$. The set-valued map $F_{0}$ corresponds to $\rho_{0}=\rho$ and $F_{1}$ to $\rho_{1}=\rho / 3$. By induction, we define a sequence of setvalued maps $F_{k}$ Lipschitzian in $x$, measurable in $t$, and such that $F_{k+1}(t, x) \subset$ $F_{k}(t, x)$ for all $(t, x) \in\left[t_{0}, t_{1}\right] \times \mathbb{R}^{n}$.

To prove the inclusion $\mathcal{S}_{\left[t_{0}, t_{1}\right]}(F) \subset \mathcal{S}_{\left[t_{0}, t_{1}\right]}\left(F_{k}\right), k \geq 0$, consider a solution $x(\cdot) \in \mathcal{S}_{\left[t_{0}, t_{1}\right]}(F)$ and the open set $x^{-1}\left(x_{r}^{k}+\rho_{k}\right.$ int $\left.B\right)=A_{r}^{k} \subset\left[t_{0}, t_{1}\right], r \in \mathbb{Z}^{n}$. If $t \in A_{r}^{k}$, then we have $\dot{x}(t) \in F(t, x(t)) \subset \operatorname{clco} F\left(t, x_{r}^{k}+2 \rho_{k} \operatorname{int} B\right)$. Set

$$
w(t)= \begin{cases}f\left(t, x_{r}^{k}\right), & t \notin A_{r}^{k} \\ \dot{x}(t), & t \in A_{r}^{k}\end{cases}
$$

where $f$ is the function from condition (C3). Since $w(t) \in G_{r}^{k}(t)$, we have $w(t) \in \Phi_{r}^{k}(t)$ for almost all $t \in\left[t_{0}, t_{1}\right]$. Consequently $\dot{x}(t) \in \Phi_{r}^{k}(t)$ for almost all $t \in A_{r}^{k}$. It is clear that $t \in A_{r}^{k}$ if and only if $x(t) \in x_{r}^{k}+\rho_{k}$ int $B$ or, equivalently, $p_{r}^{k}(x(t))>0$. Consider the set of points $t$ such that for any $r \in I_{k}^{t}=\left\{r \in \mathbb{Z}^{n} \mid p_{r}^{k}(x(t))>0\right\}$, the inclusion $\dot{x}(t) \in \Phi_{r}^{k}(t)$ is satisfied . The measure of this set is $t_{1}-t_{0}$. We have

$$
\dot{x}(t)=\sum_{r \in I_{k}^{t}} p_{r}^{k}(x(t)) \dot{x}(t) \in \sum_{r \in I_{k}^{t}} p_{r}^{k}(x(t)) \Phi_{r}^{k}(t)=\sum_{r \in \mathbb{Z}^{n}} p_{r}^{k}(x(t)) \Phi_{r}^{k}(t)=F_{k}(t, x) .
$$

The second claim of the theorem is proved.

Let us prove the third condition. Let $(t, x) \in\left[t_{0}, t_{1}\right] \times \mathbb{R}^{n}$ and $\epsilon>0$. Show that there exists $M_{t, x, \epsilon}$ such that $F_{m}(t, x) \subset F(t, x)+\epsilon B$ whenever $m>M_{t, x, \epsilon}$. Since the map $x \rightarrow F(t, x)$ is upper semi-continuous, there exists $\eta>0$ such that $F(t, y) \subset F(t, x)+\epsilon B$ whenever $y \in x+\eta B$. Consider $M$ such that $\rho_{m} \leq \eta / 3$ for all $m>M$. Let $r \in I_{m}^{x}=\left\{r \in \mathbb{Z}^{r} \mid x \in x_{i}^{m}+\rho_{m} \operatorname{int} B\right\}$ and $y \in x_{r}^{m}+2 \rho_{m}$ int $B$. If $m>M$, then we have

$$
|y-x| \leq\left|y-x_{r}^{m}\right|+\left|x_{r}^{m}-x\right| \leq 2 \rho_{m}+\rho_{m}=3 \rho_{m}<\eta .
$$

Therefore $F(t, y) \subset F(t, x)+\epsilon B$ whenever $y \in x_{r}^{m}+2 \rho_{m} \operatorname{int} B$. Consequently $G_{r}^{m}(t) \subset F(t, x)+\epsilon B$ for all $r \in I_{m}^{x}$. Since $F(t, x)$ is a convex set, we have

$$
F_{m}(t, x) \subset \sum_{r \in \mathbb{Z}^{n}} p_{r}^{m}(x) G_{r}^{m}(t) \subset F(t, x)+\epsilon B_{n} .
$$


The third claim of the theorem is proved.

Let $x_{k}(\cdot) \in \mathcal{S}_{\left[t_{0}, t_{1}\right]}\left(F_{k}\right)$ be a sequence uniformly converging to $x(\cdot)$. By construction of $F_{k}(t, x)$ and condition (C4) we have $\left|\dot{x}_{k}(t)\right| \leq b(t)$. By the Danford-Pettis theorem, without loss of generality, the sequence $\dot{x}_{k}(\cdot)$ converges in the weak topology of the space $L_{1}\left(\left[t_{0}, t_{1}\right], \mathbb{R}^{n}\right)$ to a function $v(\cdot)$. By the Mazur theorem there exists a sequences of convex combinations $\sum_{k=l}^{L} \lambda_{k} \dot{x}(\cdot)$ converging to $v(\cdot)$ in $L_{1}$-norm. Without loss of generality the sequence converges to $v(\cdot)$ for almost all $t \in\left[t_{0}, t_{1}\right]$. Fix $t$ such that $\sum_{k=l}^{L} \lambda_{k} \dot{x}(t) \rightarrow v(t)$, as $l \rightarrow \infty$. It is easy to see that the equality $x(t)=x\left(t_{0}\right)+\int_{t_{0}}^{t} v(s) d s$ holds. Let $\epsilon>0$. There exists $m$ such that $F_{m}(t, x(t)) \subset F(t, x(t))+\epsilon B_{n}$. Consider $l>m$ such that $\dot{x}(t) \in$ $\sum_{k=l}^{L} \lambda_{k} \dot{x}_{k}(t)+\epsilon B$ and $F_{m}\left(t, x_{k}(t)\right) \subset F_{m}(t, x(t))+\epsilon B$ whenever $k>l$. We have

$$
\begin{gathered}
\dot{x}(t) \in \sum_{k=l}^{L} \lambda_{k} \dot{x}_{k}(t)+\epsilon B \subset \sum_{k=l}^{L} \lambda_{k} F_{k}\left(t, x_{k}(t)\right)+\epsilon B \\
\subset \sum_{k=l}^{L} \lambda_{k} F_{m}\left(t, x_{k}(t)\right)+\epsilon B \subset \sum_{k=l}^{L} \lambda_{k} F_{m}(t, x(t))+2 \epsilon B \\
\subset F(t, x(t))+3 \epsilon B .
\end{gathered}
$$

Since $\epsilon>0$ is arbitrary and the set $F(t, x(t))$ is closed, we obtain $\dot{x}(t) \epsilon$ $F(t, x(t))$, that is, $x(\cdot) \in \mathcal{S}_{\left[t_{0}, t_{1}\right]}(F)$.

\section{Proof of Theorem 3.1}

To prove Theorem 3.1 we need a few auxiliary results. First recall the following well-known lemma.

Lemma 5.1. If $f(\cdot) \in L_{1}^{\mathrm{loc}}([0, \infty[, \mathbb{R})$ and there exists the limit

$$
\bar{f}=\lim _{T \rightarrow \infty} \frac{1}{T} \int_{0}^{T} f(t) d t
$$

then for all $t_{1}<t_{2}$ the equality

$$
\bar{f}=\lim _{\epsilon \downarrow 0} \frac{\epsilon}{t_{2}-t_{1}} \int_{t_{1} / \epsilon}^{t_{2} / \epsilon} f(t) d t
$$

holds. 
Applying Theorem 4.1 we construct a sequence of set-valued maps $F_{k}$ approximating the set-valued map $F$ and satisfying the Lipschitz condition $h\left(F_{k}(t, x), F_{k}\left(t, x^{\prime}\right)\right) \leq l_{k} b(t)\left|x-x^{\prime}\right|$, where $l_{k}$ is the Lipschitz constant of the functions $p_{r}^{k}(\cdot)$. Put $\tau=\epsilon t \in[0, T]$ and define the set-valued maps

$$
G_{\epsilon}^{k}(\tau, y)=F_{k}(\tau / \epsilon, y) \text { and } G_{0}^{k}(y)=\bar{F}_{k}(y)
$$

where

$$
\bar{F}_{k}(x)=\limsup _{\theta \uparrow 1} \limsup _{T \rightarrow \infty} \frac{1}{(1-\theta) T} \int_{\theta T}^{T} F_{k}(t, x) d t .
$$

Lemma 5.2. There exists $\epsilon_{b}>0$ such that all solutions $y(\cdot) \in \mathcal{S}_{[0, T]}\left(G_{\epsilon}^{k}, C\right)$ satisfy the Lipschitz condition $\left|y\left(\tau_{2}\right)-y\left(\tau_{1}\right)\right| \leq(1+b)\left|\tau_{2}-\tau_{1}\right|, \tau_{1}, \tau_{2} \in[0, T]$, whenever $\epsilon \in] 0, \epsilon_{b}[$.

Proof. Indeed, since $\dot{y} \in F_{k}(\tau / \epsilon, y) \subset b(\tau / \epsilon) B$, we have

$$
\begin{gathered}
\left|y\left(\tau_{2}\right)-y\left(\tau_{1}\right)\right| \leq \int_{\tau_{1}}^{\tau_{2}} b\left(\frac{\tau}{\epsilon}\right) d \tau=\epsilon \int_{\tau_{1} / \epsilon}^{\tau_{2} / \epsilon} b(s) d s \\
=\left(\tau_{2}-\tau_{1}\right) \frac{\epsilon}{\tau_{2}-\tau_{1}} \int_{\tau_{1} / \epsilon}^{\tau_{2} / \epsilon} b(s) d s .
\end{gathered}
$$

Using Condition (C5) and applying the previous lemma we obtain the result.

Now we establish the result of Theorem 3.1 for inclusions with the righthand sides $F_{k}$. Our proof is based on ideas of Krasnosel'skii and Krein [18]. Note that, since the maps $t \rightarrow F_{k}(t, x)$ are measurable, the following lemma cannot be deduced from Plotnikov's theorem [9] even if limit (5) exists.

Lemma 5.3. Given $\eta>0$, there exists $\epsilon_{0}(\eta, k)>0$ such that $\mathcal{S}_{[0, T]}\left(G_{\epsilon}^{k}, C\right) \subset$ $\mathcal{S}_{[0, T]}\left(G_{0}^{k}, C\right)+\eta \mathcal{B}$, for all $\left.\epsilon \in\right] 0, \epsilon_{0}(k, \eta)[$.

Proof. Suppose that there exist $\eta>0$, a sequence $\epsilon_{i} \downarrow 0$, and solutions $y_{i}(\cdot) \in \mathcal{S}_{[0, T]}\left(G_{\epsilon_{i}}^{k}, C\right)$ such that $y_{i}(\cdot) \notin \mathcal{S}_{[0, T]}\left(G_{0}^{k}, C\right)+\eta \mathcal{B}$. By the Arzela-Ascoli theorem we see that without loss of generality the sequence $y_{i}(\cdot)$ converges uniformly to a continuous function $y(\cdot)$. From Lemma 5.2 we obtain $\mid y_{i}\left(\tau_{2}\right)-$ $y_{i}\left(\tau_{1}\right)|\leq(1+b)| \tau_{2}-\tau_{1} \mid$. Passing to the limit as $i$ goes to infinity, we see that $y(\cdot)$ satisfies the Lipschitz condition. Therefore, $\dot{y}(\tau)$ exists almost 
everywhere in $[0, T]$. Let $\tau_{1}$ be a Lebesgue point of the function $b(\cdot)$ such that the derivative $\dot{y}\left(\tau_{1}\right)$ exists. Since $F_{k}$ is Lipschitzian, we get

$$
\begin{gathered}
y_{i}\left(\tau_{2}\right)-y_{i}\left(\tau_{1}\right) \in \int_{\tau_{1}}^{\tau_{2}}\left[F_{k}\left(\frac{s}{\epsilon_{i}}, y\left(\tau_{1}\right)\right)+l_{k} b\left(\frac{s}{\epsilon_{i}}\right)\left|y_{i}(s)-y\left(\tau_{1}\right)\right| B\right] d s \\
\subset \int_{\tau_{1}}^{\tau_{2}} F_{k}\left(\frac{s}{\epsilon_{i}}, y\left(\tau_{1}\right)\right) d s \\
+\left[\int_{\tau_{1}}^{\tau_{2}} l_{k} b\left(\frac{s}{\epsilon_{i}}\right)\left(\left|y_{i}(s)-y_{i}\left(\tau_{1}\right)\right|+\left|y_{i}\left(\tau_{1}\right)-y\left(\tau_{1}\right)\right|\right) d s\right] B .
\end{gathered}
$$

Observe that

$$
\begin{gathered}
\int_{\tau_{1}}^{\tau_{2}} l_{k} b\left(\frac{s}{\epsilon_{i}}\right)\left(\left|y_{i}(s)-y_{i}\left(\tau_{1}\right)\right|+\left|y_{i}\left(\tau_{1}\right)-y\left(\tau_{1}\right)\right|\right) d s \\
\leq l_{k}\left(\tau_{2}-\tau_{1}\right)\left[(1+b) \int_{\tau_{1}}^{\tau_{2}} b\left(\frac{s}{\epsilon_{i}}\right) d s+\frac{\left|y_{i}\left(\tau_{1}\right)-y\left(\tau_{1}\right)\right|}{\tau_{2}-\tau_{1}} \int_{\tau_{1}}^{\tau_{2}} b\left(\frac{s}{\epsilon_{i}}\right) d s\right] .
\end{gathered}
$$

Let $\tilde{\eta}>0$. If $\left|\tau_{2}-\tau_{1}\right|$ is sufficiently small and $i$ is sufficiently large, we have

$$
\begin{aligned}
& l_{k}\left[(1+b) \int_{\tau_{1}}^{\tau_{2}} b\left(\frac{s}{\epsilon_{i}}\right) d s+\frac{\left|y_{i}\left(\tau_{1}\right)-y\left(\tau_{1}\right)\right|}{\tau_{2}-\tau_{1}} \int_{\tau_{1}}^{\tau_{2}} b\left(\frac{s}{\epsilon_{i}}\right) d s\right] \\
= & l_{k}\left[(1+b)\left(\tau_{2}-\tau_{1}\right)+\left|y_{i}\left(\tau_{1}\right)-y\left(\tau_{1}\right)\right|\right] \frac{\epsilon_{i}}{\tau_{2}-\tau_{1}} \int_{\tau_{1} / \epsilon_{i}}^{\tau_{2} / \epsilon_{i}} b(\tau) d \tau<\tilde{\eta} .
\end{aligned}
$$

Therefore we obtain

$$
\frac{y_{i}\left(\tau_{2}\right)-y_{i}\left(\tau_{1}\right)}{\tau_{2}-\tau_{1}} \in \frac{\epsilon_{i}}{\tau_{2}-\tau_{1}} \int_{\tau_{1} / \epsilon_{i}}^{\tau_{2} / \epsilon_{i}} F_{k}\left(t, y\left(\tau_{1}\right)\right) d t+\tilde{\eta} B
$$

From this we get

$$
\frac{y\left(\tau_{2}\right)-y\left(\tau_{1}\right)}{\tau_{2}-\tau_{1}} \in \limsup _{T \rightarrow \infty} \frac{1}{\left(1-\tau_{1} / \tau_{2}\right) T} \int_{\left(\tau_{1} / \tau_{2}\right) T}^{T} F_{k}\left(t, y\left(\tau_{1}\right)\right) d t+\tilde{\eta} B
$$

Passing to the limit as $\tau_{2} \downarrow \tau_{1}$ we have

$$
\dot{y}\left(\tau_{1}\right) \in \bar{F}_{k}\left(y\left(\tau_{1}\right)\right)+\tilde{\eta} B
$$

Since $\tilde{\eta}$ is arbitrary we have $y(\cdot) \in \mathcal{S}_{[0, T]}\left(G_{0}^{k}, C\right)$. This implies that $y_{i}(\cdot) \in$ $\mathcal{S}_{[0, T]}\left(G_{0}^{k}, C\right)+\eta \mathcal{B}$ whenever $i$ is sufficiently large. We obtain a contradiction and the end of the proof. 
Note that in this lemma the map $F_{k}$ is Lipschitzian with a constant satisfying condition (C5). Without this condition the lemma is not true, as we can see from Example 2.3.

Lemma 5.4. If a sequence of solutions $x_{k}(\cdot) \in \mathcal{S}_{[0, T]}\left(\bar{F}_{k}, C\right)$, converges uniformly to $x(\cdot)$, then the inclusion $x(\cdot) \in \mathcal{S}_{[0, T]}(\bar{F}, C)$ holds.

Proof. Let $\delta>0$. Obviously we have $\bar{F}_{k}(x) \subset \bar{F}^{\delta}(x)$, for all $k>K(x, \delta)$. Arguing as in the prove of the last part of Theorem 4.1, we see that, given $\eta>0$, for almost all $t$ there exist positive integers $l$ and $L$, and nonnegative numbers $\lambda_{k}$ such that $\sum_{k} \lambda_{k}=1$ and the following inclusions hold:

$$
\begin{gathered}
\dot{x}(t) \in \sum_{k=l}^{L} \lambda_{k} \dot{x}_{k}(t)+\eta B \subset \sum_{k=l}^{L} \lambda_{k} \bar{F}_{k}\left(x_{k}(t)\right)+\eta B \\
\subset \sum_{k=l}^{L} \lambda_{k} \bar{F}_{l}\left(x_{k}(t)\right)+\eta B \subset \sum_{k=l}^{L} \lambda_{k} \bar{F}^{\delta}(x(t))+\eta B \\
=\bar{F}^{\delta}(x(t))+\eta B .
\end{gathered}
$$

Since $\eta>0$ is arbitrary, we have $\dot{x}(t) \in \bar{F}^{\delta}(x(t))$ for all $\delta>0$. Therefore $x(\cdot) \in \mathcal{S}_{[0, T]}(\bar{F}, C)$

Now we are in a position to prove Theorem 3.1 .

Proof of Theorem 3.1. Suppose that there exist $\eta>0$, a sequence $\epsilon_{j} \downarrow 0$, and solutions $y_{\epsilon_{j}}(\cdot) \in \mathcal{S}_{[0, T]}\left(G_{\epsilon_{j}}, C\right)$ satisfying $y_{\epsilon_{j}}(\cdot) \notin \mathcal{S}_{[0, T]}\left(G_{0}, C\right)+2 \eta \mathcal{B}$. Since

$$
\mathcal{S}_{[0, T]}\left(G_{\epsilon_{j}}, C\right) \subset \mathcal{S}_{[0, T]}\left(G_{\epsilon_{j}}^{k}, C\right),
$$

we have $y_{\epsilon_{j}}(\cdot) \in \mathcal{S}_{[0, T]}\left(G_{\epsilon_{j}}^{k}, C\right)$. From Lemma 5.3 we see that for any $k$ there exists $j_{k}$ such that

$$
y_{\epsilon_{j_{k}}}(\cdot) \in \mathcal{S}_{[0, T]}\left(G_{0}^{k}, C\right)+\eta \mathcal{B}
$$

Thus the functions $y_{\epsilon_{j_{k}}}(\cdot)$ can be represented in the form $y_{\epsilon_{j_{k}}}(\cdot)=z_{\epsilon_{j_{k}}}(\cdot)+$ $\xi_{j_{k}}(\cdot)$, where $z_{\epsilon_{j_{k}}}(\cdot) \in \mathcal{S}_{[0, T]}\left(G_{0}^{k}, C\right)$ and $\xi_{j_{k}}(\cdot) \in \eta \mathcal{B}$. By the Arzela-Ascoli theorem without loss of generality the sequences $y_{\epsilon_{j_{k}}}(\cdot)$ and $z_{\epsilon_{j_{k}}}(\cdot)$ uniformly converge to functions $y(\cdot)$ and $z(\cdot)$, respectively. This implies that the sequence $\xi_{j_{k}}(\cdot)$ uniformly converges to a function $\xi(\cdot) \in \eta \mathcal{B}$. By Lemma 5.4 we have

$$
y(\cdot) \in \mathcal{S}_{[0, T]}\left(G_{0}, C\right) .
$$

Hence $y_{\epsilon_{j_{k}}}(\cdot) \in \mathcal{S}_{[0, T]}\left(G_{0}, C\right)+2 \eta \mathcal{B}$ whenever $k$ is large enough. This contradiction proves the theorem. 


\section{Proof of Theorem 3.2}

Assume that $y=0$ is an asymptotically stable equilibrium position of the averaged differential inclusion $\dot{y} \in G_{0}(y)$. The following lemma is an immediate consequence of Lemma 1 from [4, Sec. 15].

Lemma 6.1. For any $\eta>0$ there exist $\delta \in] 0, \eta[$ and $T>0$ such that all solutions $\bar{y}(\cdot) \in \mathcal{S}_{[0, T]}\left(G_{0}, \delta B\right)$ satisfy the inclusions $\bar{y}(\cdot) \in(\eta / 2) \mathcal{B}$ and $\bar{y}(T) \in(\delta / 2) B$.

The proof of Theorem 3.2 is based on this lemma and Theorem 3.1 .

Proof of Theorem 3.2. Let $\eta>0$. Applying Lemma 6.1 we construct $\delta>$ 0 and $T>0$. From Theorem 3.1, we see that there exists $\epsilon_{0}$ such that for any solution $\left.y(\cdot) \in \mathcal{S}_{[0, T]}\left(G_{\epsilon}, \delta B\right), \epsilon \in\right] 0, \epsilon_{0}[$, there is a solution $\bar{y}(\cdot) \in$ $\mathcal{S}_{[0, T]}\left(G_{0}, \delta B\right)$ satisfying the inclusion $y(\cdot) \in \bar{y}(\cdot)+(\delta / 2) \mathcal{B}$. Since $\bar{y}(\cdot) \in$ $(\eta / 2) \mathcal{B}$ and $\bar{y}(T) \in(\delta / 2) B$, we have $\bar{y}(\cdot) \in(\eta / 2+\delta / 2) \mathcal{B} \subset \eta \mathcal{B}$ and $y(T) \in \delta B$. Applying this construction on the intervals $[T, 2 T]$, $[2 T, 3 T]$, etc., we obtain the result.

To illustrate Theorem 3.2 consider the following example.

\subsection{Example}

Let $x=\left(x_{1}, x_{2}\right) \in \mathbb{R}^{2}$. Consider the function

$$
f_{0}(x)= \begin{cases}(1,-1), & x_{1}>0, x_{2}>0 \\ (-1,1 / 2), & x_{1}>0, x_{2}<0 \\ (-1,1), & x_{1}<0, x_{2}<0 \\ (1,-1 / 2), & x_{1}<0, x_{2}>0\end{cases}
$$

and define the set-valued maps $F_{0}(x)=\bigcap_{\delta>0} \operatorname{clco} f_{0}(x+\delta B)$ and $F(t, x)=$ $F_{0}(x)+\{(\sin t, \cos t)\}$. It is easy to see that $\bar{F}(x)=F_{0}(x)$ and $0 \in \bar{F}(0)$. The zero equilibrium position of the averaged differential inclusion is asymptotically stable. By Theorem 3.2 the solutions of the differential inclusions $\dot{x} \in \epsilon F(t, x)$ are close to zero for all $t \geq 0$, whenever $\epsilon>0$ is sufficiently small. 


\section{Acknowledgements}

The authors are grateful to the anonymous referee for his/her valuable suggestions, to Vasile Staicu and Vladimir Goncharov for a fruitful discussion of this work, and to Andrzej Nowak for bibliographical support. This research was supported by the Portuguese Foundation for Science and Technologies (FCT), the Portuguese Operational Programme for Competitiveness Factors (COMPETE), the Portuguese National Strategic Reference Framework (QREN), and the European Regional Development Fund (FEDER).

\section{References}

[1] N. N. Bogoliubov, Y. A. Mitropolski, Asymptotic Methods in the Theory of Non-Linear Oscillations, Gordon and Breach, New York, 1961.

[2] A. M. Samoilenko, A. N. Stanzhitskii, On the averaging of differential equations on an infinite interval, Differential Equations, Vol. 42, Number 4, (2006), pp 476-482.

[3] A. D. Guerman, M. Yu. Ovchinnikov, V. I. Penkov, V. A. Sarychev. Nonresonant motions of a satellite with hysteresis rods under conditions of gravity orientation. Mechanics of Solids, Vol. 24, (1989), pp 1 - 11.

[4] A. F. Filippov, Differential Equations with Discontinuous Right-hand Sides, Mathematics and Its Applications, Vol. 18 - Kluer Academic Publishers (1988).

[5] G. V. Smirnov, Introduction to the Theory of Differential Inclusions, AMS Graduate Studies in Mathematics, Vol. 41 (2002).

[6] M. Moussaoui, Approximations Lipschitziennes de multifonctions. Application aux conditions nćessaires d'optimalité, Sémin. Anal. Convexe, Univ. Sci. Tech. Languedoc 20, (1990), Exp. No.6, 34 pp.

[7] A. Kucia, A. Nowak, Some results on Caratheodory type multifunctions, Vol. 51, (2003), pp 283-290.

[8] G. V. Smirnov, Extremal Problems for Differential Inclusions, PhD Thesis, Moscow Institute of Physics and Technology, 1987 (in Russian). 
[9] V. A. Plotnikov, Averaging method for differential inclusions and its application to optimal control problems, Differential Equations, Vol. 15, (1979), pp 1013-1018.

[10] V. A. Plotnikov, On averaging of differential inclusions in the case where average of the right-hand side does not exist, Journal of Mathematical Sciences, Vol. 80, Number 1, (1996), pp 1779-1784.

[11] V. A. Plotnikov, Asymptotic methods in the theory of differential equations with discontinuous and multi-valued right-hand sides, Ukranian Mathematical Journal, Vol. 48, Number 11, (1996), pp 1605-1616.

[12] N. V. Plotnikova, The Krasnosel'skii-Krein theorem for differential inclusions, Differential Equations, Vol. 41, Number 7, (2005), pp 10491053.

[13] G. Grammel, Averaging of set-valued differential equations, International Journal of Mathematics and Mathematical Sciences, (2003), Issue 25, pp 1615-1622.

[14] T. Donchev, G. Grammel, Averaging of differential inclusions, Journal of Mathematical Analysis and Applications, Vol. 311, (2005), pp 402416.

[15] V. S. Klimov, Averaging of differential inclusions, Differential Equations, Volume 44, Number 12, (2008), pp 1673-1681.

[16] M. Lakrib, An averaging theorem for ordinary differential inclusions, Bull. Belg. Math. Soc. Simon Stevin Vol. 16, (2009), pp 1329.

[17] G. Haddad Topological properties of the set of solutions for functional differential inclusions. Nonlinear Analysis, 1981, Vol. 5, pp 1349-1366.

[18] M. A. Krasnosel'skii, S. G. Krein, On the principle of averaging in nonlinear mechanics, (Russian) Uspehi Mat. Nauk (N.S.) Vol. 10, Number 3 (65), (1955), pp 147-152.

[19] A. A. Tolstonogov, Differential Inclusions in a Banach Space, Mathematics and Its Applications, Vol. 524 - Kluer Academic Publishers (2000). 